\title{
Characterization of synthesized NANO-encapsulated drug for bone loss on hind limb suspension rat model by NMR and micro-CT
}

\author{
Qingwen $\mathrm{Ni}^{1,2 *}$, Hong Dixon ${ }^{1}$, Gloria Gutierrez ${ }^{1}$, Long $\mathrm{Bi}^{3}$, Yi-Xian Qin $^{3}$ \\ ${ }^{1}$ Southwest Research Institute, San Antonio, TX, USA \\ ${ }^{2}$ Texas A\&M International University, Laredo, TX, USA \\ ${ }^{3}$ State University of New York at Stony Brook, Stony Brook NY, USA
}

Email address:

qni@tamiu.edu (Qingwen Ni), qni@swri.org (Qingwen Ni)

\section{To cite this article:}

Qingwen Ni, Hong Dixon, Gloria Gutierrez, Long Bi, Yi-Xian Qin. Characterization of Synthesized NANO-encapsulated Drug for Bone Loss on Hind Limb Suspension Rat Model by NMR and Micro-CT. Advances in Bioscience and Bioengineering. Vol. 1, No. 1, 2013; pp. 1-7. doi: $10.11648 /$ j.abb.20130101.11

\begin{abstract}
A formulation of nano-encapsulated enantiomer of $(+)$ promethazine with desired release rate has been synthesized for establish a localized drug delivery system. It was tested on a hind limb suspension (HLS) disuse rat model, and by using a non-destructive Nuclear Magnetic Resonance (NMR) relaxation technique, and micro computed tomography (Micro-CT) analysis technique to qualitatively evaluate the effectiveness of the new bone formations as well as to compare the current commercial anti-bone loss drug Alendeonate. Our studies suggest that nano-encapsulated $(+)$ promethazine in controlled release formulations conjugating bone-targeting functional groups are effective in promoting bone growth in a disuse rat model.
\end{abstract}

Keywords: Nano-Encapsulation, Bone, NMR, Micro-CT

\section{Introduction}

Bone loss, osteoporosis, is recognized as a significant major public health problems as well as in the space programs worldwide. Osteopenia is a disease characterized by long term loss of bone tissue, particularly in the weight-supporting skeleton [1-2]. Results of the joint Russian/US studies on the effect of microgravity on bone tissue from 4.5- to 14.5-month long missions have demonstrated that bone mineral density (BMD, $\left.\mathrm{g} / \mathrm{cm}^{2}\right)$ and mineral content (BMC, g) are diminished in all areas of the astronaut skeleton [3]. While osteopenia can affect the whole body, complications often occur predominantly at specific sites of the skeleton with great load bearing demands The greatest BMD losses have been observed in the skeleton of the lower body, i.e., in pelvic bones $(-11.99 \pm 1.22 \%)$ and in the femoral neck $(-8.17 \pm 1.24 \%)$, while there was no apparent decay found in the skull region. On average, the magnitude and rate of the loss is staggering; astronauts lose bone mineral in the lower appendicular skeleton at a rate approaching $2 \%$ per month [4-5]. Similar results were found in the bed rest study. In a -6 degrees head-down tilt 7-day bed rest model for microgravity, it was observed that there was a decreased bone formation rate in the iliac crest [6]. To effectively countermeasure the bone loss, we need a better therapeutic system that can deliver the treatment in a need-base and non-invasively. An adequate understanding of the underlying mechanism and treatment strategy of such skeletal complications are extremely needed.

Most bone tissue turnover occurs at bone surfaces, such as at the interface to the marrow or in Haversian systems. Bone surfaces are normally covered by lining cells. In response to resorption stimuli these lining cells retract and expose the bone surface to attachment by osteoclasts and subsequent bone turnover. Therefore, targeting to the calcified matrix is most likely to occur at sites of active resorption. Bisphosphonates exhibit exceptionally high affinity to bone mineral hydroxyapatite; using a inactive bisphosphonate moiety deliver NANO-encapsulated $(+)$ promethazine provide a promising method to treat a variety of bone diseases [7-12]. Promethazine, an $\mathrm{H}_{1}$ receptor blocker phenothiazine, was found to inhibit age-related bone loss in animal studies [13]; and the $(+)$ enantiomer of promethazine was found to have a threefold higher efficacy 
for osteoclast inhibition than both the racemate and the (-) enantiomer [14].

The objective of this research study is to establish a unique localized drug delivery system for bone loss in the critical region by developing a NANO-encapsulated medicine protocol to accelerate local therapeutic effects, and by using a non-invasive NMR relaxation technique, and micro-CT analysis technique to evaluate the effectiveness of the formation as well as to compare the current commercial anti-bone loss drug Alendeonate. In this program we have developed effective, less toxic NANO-encapsulated drug, and control the release rate of NANO-encapsulated formation to prevent bone loss by preparation of enantiomers of promethazine, and employ an animal (rat) disuse model to determine the efficacy of the bone-targeting micro-nanocapsule formulations of promethazine.

\section{Experimental Section}

\subsection{Animal Model and Sample Population}

A disuse animal model is developed through generated reduced or zero lower limb weight-bearing disuse hind limb suspension (HLS) rat model with age of 5 months [24]. Rat femurs obtained from the Department of Biomedical Engineering, SUNY Stony Brook, New York through a collaborative relationship between SUNY and Southwest Research Institute (SwRI). Animal experiment was guided by the IACUC. HLS preparations were initially performed on the two tests. In each test the adaptive responses were evaluated following four weeks period on 6 month female rats. The test was applied on total of 6 groups $(\mathrm{N}=5$ per group) in female rats: 1) disuse sham, 2) disuse plus drug of 035-P18, 3) disuse plus drug of 035-P21, 4) disuse plus Alendronate, 5) age match normal control, and 6) baseline control (animals' bone harvested at the beginning of the experimental period) (Table 2). A preliminary study (test 1) was performed prior to this reported study, in which the dosage of the drug was at $0.33 \mathrm{mg} / \mathrm{kg}$ body weight. The results showed less effective and insignificant. Based on this preliminary test, the drug dosage of $0.66 \mathrm{mg} / \mathrm{kg}$ body weight (every two days, IP injection) was selected in the second test reported in this study. All the samples (right legs) were cleaned of soft tissues, and wrapped in calcium gauze and stored in separate containers filled with calcium buffered saline (CBS) and frozen at approximately $-20^{\circ} \mathrm{C}$ until testing.

\subsection{NMR Determination}

It is known that Nuclear Magnetic Resonance (NMR) proton spin-spin (T2) or spin-lattice (T1) relaxation time measurements and analytical processing techniques have been used to determine microstructural characteristics of various types of fluid filled porous materials with characteristic pore sizes ranging from sub-micron to sub-millimeter [15-18]. Currently this method has been developed and applied to quantify on human cortical bone [19-23]. The observed proton NMR relaxation signals are a convolution of the relaxation of fluid in the pores throughout the observed system with the longer relaxation time corresponding to larger pore sizes [27].

\subsubsection{NMR Measurement}

A home-built 0.5 to $40 \mathrm{MHz}$ broadline NMR system with an electromagnet of 19 inch diameter with a 4 inch gap was set up for a proton frequency of $27 \mathrm{MHz}$ for these measurements. A laboratory-built 1.0 inch diameter rf coil was used in the experiment.1H spin-spin (T2) relaxation profiles were obtained by using NMR CPMG [25-26] $\{900$ $[-\tau-1800-\tau$ (echo)]n - TR $\}$ spin echo method with a 6.5 $\mu$ s wide $90 \mathrm{o}$ pulse, $\tau$ of $500 \mu \mathrm{s}$, and TR (sequences repetition rate) of $15 \mathrm{~s}$. Each $\mathrm{T} 2$ profile, one thousand echoes (one scan with $n=1000$ ) were acquired and forty scans were used. Thus, one scan has repeated 1000 echoes in the window. The data was measured from fresh frozen rat bone after complete thawing in the room temperature $(21 \pm 10 C)$.

\subsubsection{The Relationship between NMR Data and Effective Pore Sizes}

Based on the low field NMR principle the diffusion effect may be negligible. Here, we accept the Brownstein and Tarr assumption [27] that the relaxation rate $1 / T_{2}$ is proportional to the surface-to-volume $(\mathrm{S} / \mathrm{V})$ ratio of the pore

$$
1 / T 2=\rho(S / V)_{\text {pore }}
$$

where $\rho$ is the surface relaxivity, which is a measure of the effects of the pore surface enhancing the relaxation rate. Equation (1) indicates that the NMR relaxation time is proportional to pore size.

For a porous bone, the observed NMR magnetization will depend upon the $T_{2}$ of broad distributions of water in all pores. This implies that NMR transverse relaxation $\left(T_{2}\right)$ data can be expressed as a sum of exponential functions:

$$
M\left(t_{i}\right)=\sum_{j=1}^{m} f\left(T_{2, j}\right) \exp \left(-t_{i} / T_{2, j}\right)
$$

where $f\left(T_{2, j}\right)$ is proportional to the number of spins which relax with a time constant $T_{2, j} . M\left(t_{i}\right)$ is the NMR magnetization decay from fluid saturated cortical bone. Equation (2) can be inverted into a $T_{2}$ relaxation time distribution. Thus, instead of estimating a single relaxation time from a magnetization decay, it is necessary to estimate a inversion $T_{2}$ spectrum or distribution of relaxation time $\mathrm{f}\left(\mathrm{T}_{2, \mathrm{j}}\right)$, and an inversion relaxation technique was applied $[15-16,19,20,23]$. Since $\mathrm{T}_{2}$ depends linearly upon pore size, the $\mathrm{T}_{2}$ distribution corresponds to pore-size distribution with the longer relaxation times having the larger pores. In addition, the median $T_{2}$ from the $T_{2}$ relaxation distribution can provide the overall effective bone pore size, i.e., the quality of whole bone. These results 
are also compared with microCT data.

\subsubsection{Median T2 Relaxation Time}

The median T2 relaxation calculation is based on $\mathrm{T}_{2}$ relaxation distribution data. In $\mathrm{T}_{2}$ relaxation distribution spectra, the water intensity (amplitude in y axis) is plotted against $\mathrm{T}_{2}$ relaxation time (x-axis) which corresponds to different pore sizes and the cumulative water intensity amplitudes, and is normalized to 1.Therefore, the middle point 0.5 on y axis corresponds to the median relaxation time on $\mathrm{x}$-axis (Figure 3 ). This median relaxation time method can provide the relaxation mechanism for whole bone without considering the bone size difference, i.e. different bone volume for different bone.

\subsection{MicroCT Analysis}

A high resolution microCT scanner (microCT-40, SCANCO Medical AG, Bassersdorf, Switzerland) was used, and the samples were scanned with a spatial resolution of 15 $\mu \mathrm{m}$. In basic bone research bone volume density (BV/TV), trabecular spacing (Tb. Sp), and trabecular number (Tb.N) are key measurements characterizing the three-dimensional (3D) structure of bone by microCT technology. BV/TV indicates the fraction of a given volume of interest (VOI, i.e. the Total Volume TV) that is occupied by mineralized bone (Bone Volume). BV/TV is usually reported as a \% value and it can be used to evaluate relative changes in bone volume density following a given treatment. One way to determine the efficacy of the drug is to compare BV/TV in defined VOIs in control (or negative control group) vs treatment group. You would expect that if the drug works, the treatment group shows higher BV/TV values than the HLS group. Trabecular separation (Tb.Sp.mm) is essentially the thickness of the spaces as defined by binarisation within the VOI. It can also be calculated wither from $2 \mathrm{D}$ images with model assumptions or directly in 3D. Trabecular number (Tb.N. 1/mm) implies the number of traversals across a trabecular or solid structure made per unit length on a linear path through a trabecular bone region. The concept for measuring the average trabecular the average separation and average number can be applied to any structure and used in many different fields.

\section{Results and Discussion}

\subsection{Synthesis of PLGA Nanoparticles with Encapsulated (+) Promethazine}

From our study the nanoparticles of $(+)$ promethazine in block copolymers of poly (ethylene glycol)-b-poly(lactic acid glycolic acid) (PEG-PLGA) PLGA were successfully prepared with about $2 \%$ payload and an encapsulation efficiency of $40 \%$ by a double emulsion method [28]. The nanoparticle size distributions are shown in Figure 1. The positively charged nanoparticle samples demonstrated controlled release of $(+)$ promethazine for a day (Figure 2).The lyophilized nanoparticles can be re-suspended in
pH7.4 PBS.

Three nanoparticle samples were prepared for in vivo testing. Two encapsulated drugs with different concentrations of $(+) \mathrm{PMZ}$, and one with pure Alendronate sodium were successfully synthesized. The details of the drug samples are shown in Table 1 below.

After the data analysis from initial test (drug dose 0.33 $\mathrm{mg}$ per rat body weight) it was selected the drug dose $(0.66$ $\mathrm{mg} / \mathrm{kg}$ ) for the following test protocol in Table 2 on second test.

\subsection{NMR Results}

It is found that the median $\mathrm{T} 2$ relaxation time as measured by NMR is one of the best parameters for whole bone (includes cortical, trabecular, and mallow) porosity evaluation, it can be used to effectively determine bone quality changes under various testing conditions for the animals (e.g. HLS, HLS + drug, and normal only). The median $\mathrm{T} 2$ relaxation calculation is based on $\mathrm{T} 2$ relaxation distribution data. The middle point 0.5 on $y$ axis corresponds to the median relaxation time on $\mathrm{x}$-axis (Figure 3). This median relaxation time method can provide the whole relaxation mechanism without considering the bone size difference, i.e. different bone volume for different bone. It is also a sensitive method to analyze all pore size changes in an entire bone.

In figure 3 , the longer relaxation time corresponds tolarger pore sizes and the higher intensity corresponds to larger pore volume. Therefore, it is assumed that the first peak (from left to right) mainly is contributed from the cortical bone region, the second peak is mainly contributed from cortical bone and bone marrow, and the third peak is mainly contributed from the trabecular region.

A summary of the median $\mathrm{T} 2$ relaxation times obtained from the in vivo animal studies (at normal, HLS only, HLS+drug alendronate, HLS+drug 035-P18, and HLS+drug 035-p21 are listed in Table 3. It is suggested that on average the drug 035-p18 improves significantly over the disuse HLS with better than drug 035-p21, and slightly better than the currently in commercial used alendronate.

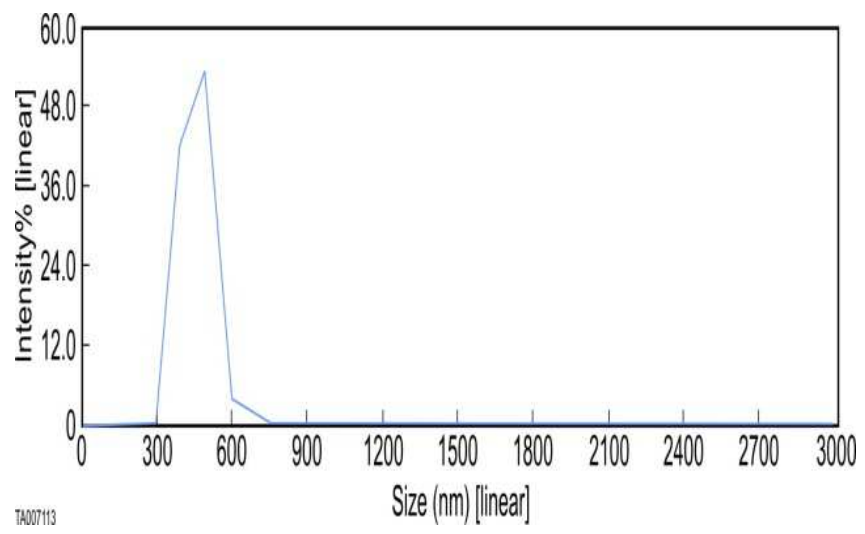

Figure 1. Particle size distribution of prepared (+)promethazine/PLGA nanoparticles 
Cumulative promethazine release (in pH 7.4 PBS at $37^{\circ} \mathrm{C}$ ) Sample 07-0203-058-p39

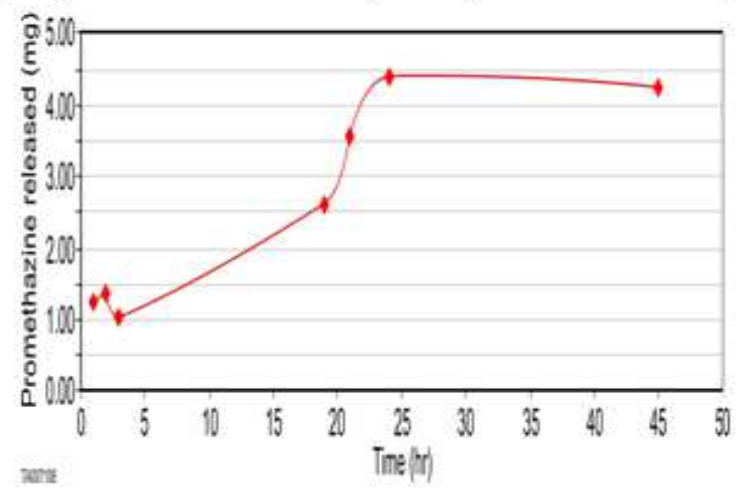

Figure 2. In vitro test for controlled release of nanoparticles of (+)promethazine
Table 1. Sample Detail

\begin{tabular}{|c|c|c|}
\hline Sample No. & Weight(mg) & Composition \\
\hline 10-0203-035-p18 & 481 & $\begin{array}{l}\text { 14\%(+)PMZ/86\% } \\
\text { PLGA-10\%PEG }\end{array}$ \\
\hline $10-0203-035-\mathrm{p} 21$ & 632 & $\begin{array}{l}40 \%(+) \mathrm{PMZ} / 60 \% \\
\text { PLGA-10\%PEG }\end{array}$ \\
\hline $\begin{array}{l}\text { Alendronate } \\
(\mathrm{ALN})\end{array}$ & 500 & $\begin{array}{l}100 \% \text { Alendronate } \\
\text { sodium trihydrate }\end{array}$ \\
\hline
\end{tabular}

Table 2. Test Protocol

\begin{tabular}{lll}
\hline Group & Group Description & Animals \\
\hline 1 & Age-Matched Control Only & 5 \\
2 & Hind-limb Suspension Only & 5 \\
3 & Hind-limb Suspension + DRUG (035-p18) & 5 \\
4 & Hind-limb Suspension + DRUG (035-p21) & 5 \\
5 & Hind-limb Suspension + Alendronate (ALN) & 5 \\
6 & Baseline Control (bone samples harvested at day 0) & 5 \\
\hline
\end{tabular}
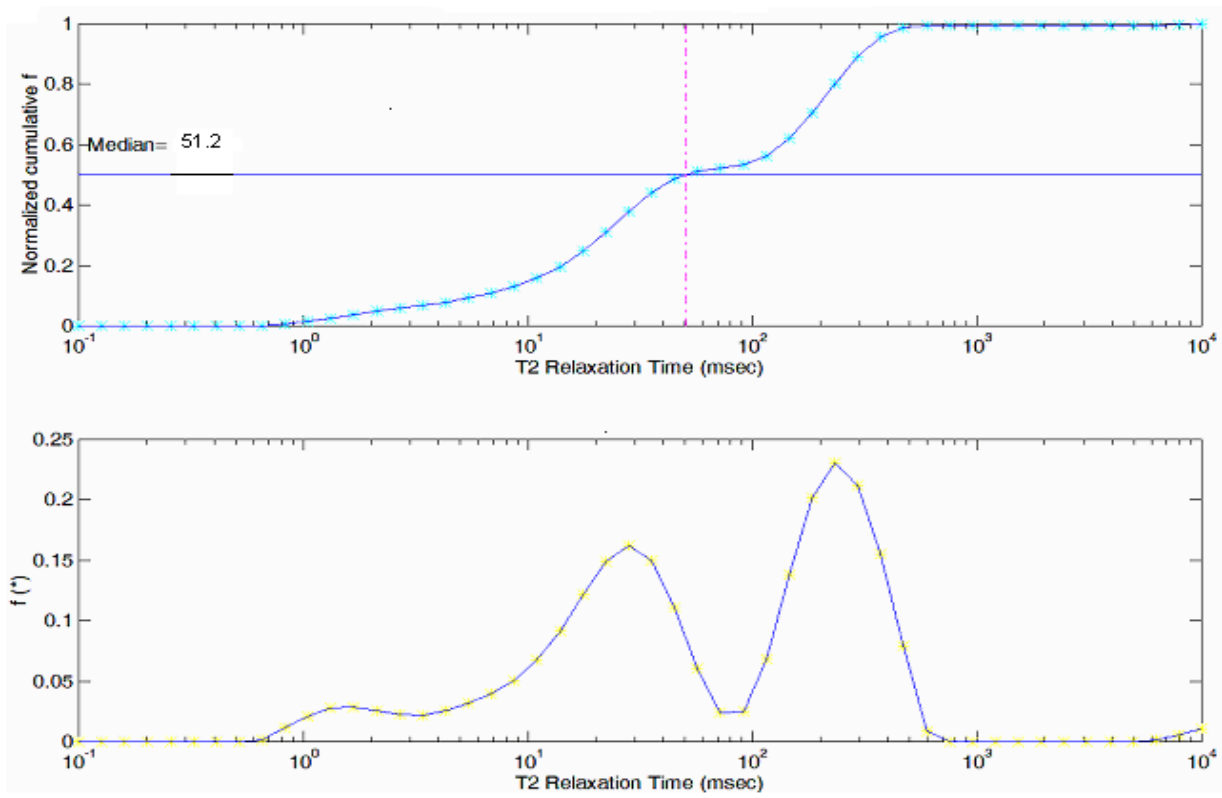

Figure 3. NMR T2 relaxation time distribution and median T2 relaxation for sample A10 (normal).

Table 3. Median Relaxation Times listed for Different Group Samples

\begin{tabular}{|c|c|c|c|c|c|}
\hline $\begin{array}{l}\text { Sample \# } \\
\text { (Normal) }\end{array}$ & $\begin{array}{l}\text { Median } \\
\text { relaxation }(\mathrm{ms})\end{array}$ & $\begin{array}{l}\text { Sample \# } \\
\text { (HLS) }\end{array}$ & $\begin{array}{l}\text { Median } \\
\text { relaxation }(\mathrm{ms})\end{array}$ & $\begin{array}{l}\text { Sample \# } \\
\text { (HLS+ } \\
\text { Alendronate) }\end{array}$ & $\begin{array}{c}\text { Median relaxation } \\
(\mathrm{ms})\end{array}$ \\
\hline A6 & 56.80 & A11 & 56.21 & A20 & 56.26 \\
\hline A7 & 57.68 & A12 & 61.43 & A21 & 51.70 \\
\hline A8 & 48.64 & A13 & 59.82 & A22 & 54.97 \\
\hline A9 & 53.36 & A14 & 61.88 & A23 & 54.56 \\
\hline A10 & 51.20 & A 15 & 58.58 & A24 & 58.40 \\
\hline Average & $53.54 \pm 3.78$ & & $59.58 \pm 2.30$ & & $55.18 \pm 2.45$ \\
\hline $\begin{array}{l}\text { Sample \# } \\
\text { (HLS+drug 035-P18) }\end{array}$ & $\begin{array}{l}\text { Median } \\
\text { relaxation }(\mathrm{ms})\end{array}$ & $\begin{array}{l}\text { Sample \# } \\
\text { (HLS+drug } \\
\text { 035-P21) }\end{array}$ & $\begin{array}{l}\text { Median } \\
\text { relaxation (ms) }\end{array}$ & & \\
\hline A26 & 54.94 & A32 & 54.44 & & \\
\hline $\mathrm{A} 27$ & 55.20 & A33 & 58.03 & & \\
\hline A 28 & 49.44 & A34 & 60.62 & & \\
\hline A29 & 51.84 & A35 & 59.93 & & \\
\hline A30 & 54.62 & A36 & 56.42 & & \\
\hline Average & $53.21 \pm 2.50$ & & $57.88 \pm 2.53$ & & \\
\hline
\end{tabular}




\subsection{Micro-CT Results}

Throughout the entire experimental period, the animals' body weight was monitored. The body weights were not significantly different between groups at the beginning of the study, with an average of $320 \pm 47 \mathrm{~g}$.

From the data below, the lack of weight-bearing activity for 4 weeks significantly reduced trabecular bone quality and quantity, in the distal metaphyseal region directly above the growth plate demonstrated by a $34.7 \%$ decreases in the average of $\mathrm{BV} / \mathrm{TV}$, a $18.7 \%$ decreases in the average of Tb.N, and a $28.2 \%$ increases in the average of $\mathrm{Tb} . \mathrm{Sb}$ comparing between HLS and No HLS control groups. Summaries of microCT measurements are in the following Tables and corresponding Figures 4-6. Here, in Tables the columns 1 to 6 are counted from left to right corresponding to the relative Figures.

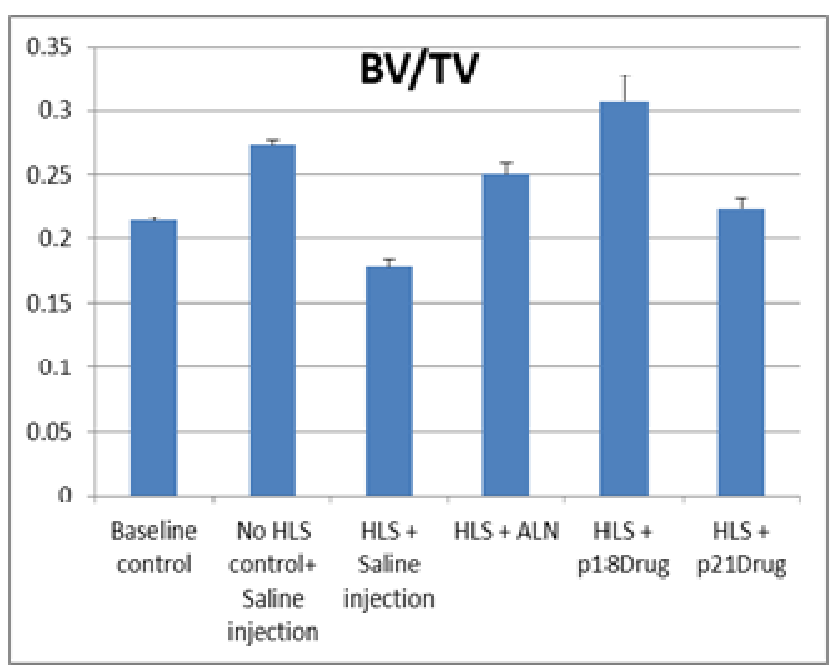

Figure 4. Bone volume fraction (BV/TV) changes among control, HLS and HLS plus different drugs.

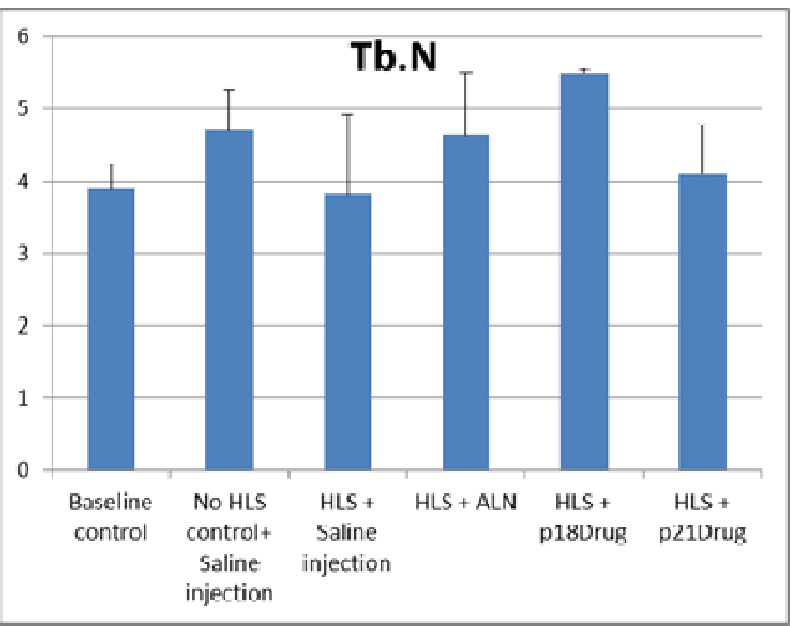

Figure 5. Bone trabecular number (Tb.N, 1/mm) changes among control, HLS and HLS plus different drugs.

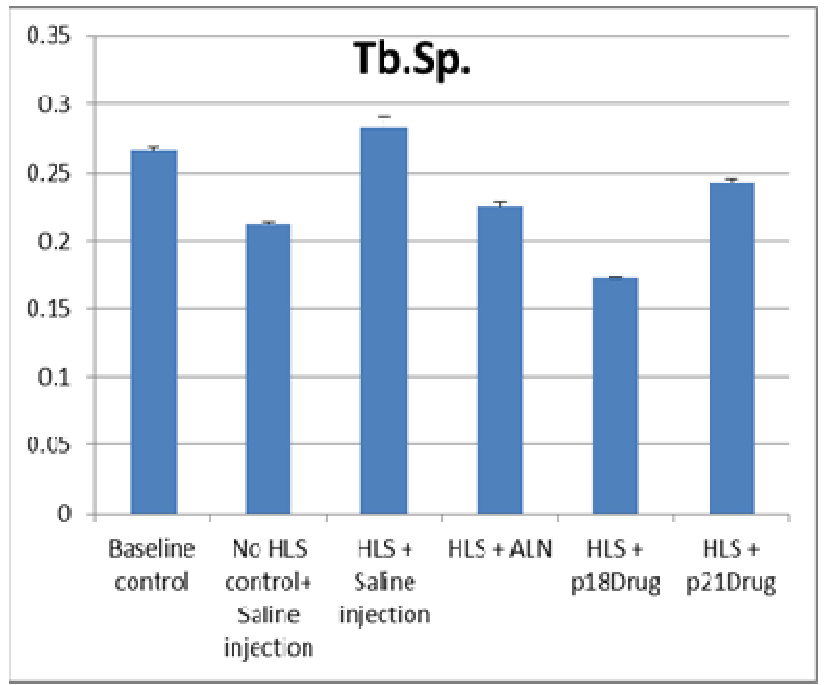

Figure 6. Bone trabecular separation (Tb.Sp, mm) changes among control, HLS and HLS plus different drugs.

Table 4. Summary $(B V / T V)$

\begin{tabular}{lllll}
\hline Groups & Count & Sum & Average & Variance \\
\hline Column 1 & 5 & 0.8552 & 0.2138 & 0.002748 \\
Column 2 & 5 & 1.3655 & 0.2731 & 0.003962 \\
Column 3 & 5 & 1.4260 & 0.1783 & 0.005160 \\
Column 4 & 5 & 1.0017 & 0.2504 & 0.008963 \\
Column 5 & 5 & 1.5320 & 0.3064 & 0.02034 \\
Column 6 & 5 & 1.1219 & 0.2244 & 0.007890 \\
\hline
\end{tabular}

Table 5. Summary (Tb.N)

\begin{tabular}{lllll}
\hline Groups & Count & Sum & Average & Variance \\
\hline Column 1 & 5 & 15.5490 & 3.8873 & 0.3399 \\
Column 2 & 5 & 23.5018 & 4.7004 & 0.5415 \\
Column 3 & 5 & 30.5813 & 3.8227 & 1.0944 \\
Column 4 & 5 & 18.5307 & 4.6327 & 0.8616 \\
Column 5 & 5 & 21.9777 & 5.4944 & 0.0444 \\
Column 6 & 5 & 20.5668 & 4.11336 & 0.6592 \\
\hline
\end{tabular}

Table 6. Summary (Tb. Sp)

\begin{tabular}{lllll}
\hline Groups & Count & Sum & Average & Variance \\
\hline Column 1 & 5 & 1.0643 & 0.2661 & 0.002490 \\
Column 2 & 5 & 1.0604 & 0.2121 & 0.001110 \\
Column 3 & 5 & 2.2596 & 0.2825 & 0.007691 \\
Column 4 & 5 & 0.8992 & 0.2248 & 0.002380 \\
Column 5 & 5 & 0.6891 & 0.1723 & $2.080 \mathrm{E}-05$ \\
Column 6 & 5 & 1.2174 & 0.2435 & 0.002618 \\
\hline
\end{tabular}

\section{Conclusions}

The results from our studies are positive. It is suggested that nano-encapsulated $(+)$ promethazine in controlled release formulations conjugating bone-targeting functional groups can be effective in anti-bone loss in a HLS rat model. The improvement is quite significant, especially, for the drug sample of 035-p18. The test results show that after 
the treatments on HLS group, the average bone porosity values were approachable or better than those of the control (normal) groups. It was demonstrated the $(+) \mathrm{PMZ}$ can be functional as anti-bone loss at microgravity condition and other similar conditions. During the experiment a preliminary test (test 1 ) was performed prior to this reported study, in which the dosage of the drug was at $0.33 \mathrm{mg} / \mathrm{kg}$ body weight and the results showed the changes among HLS and HLS+drugs (HLS+alendronate, HLS+drug 035-p18, and HLS+035-p21) less effective and insignificant. Based on this preliminary test, the drug dosage of $0.66 \mathrm{mg} / \mathrm{kg}$ body weight was selected in the second test reported in this study. This dosage selection is according to Promethazine for human oral is $25-50 \mathrm{mg}$ per time, and for per $\mathrm{Kg}$ mass is $25-50 \mathrm{mg} / 75 \mathrm{~kg}=0.33-0.66 \mathrm{mg} / \mathrm{kg}$. Therefore, the certain amount of drug dosage is needed to insure anti-bone loss function of the drug.

The second HLS test showed a very positive result as we reported, i.e. the significant function of anti-bone loss. The results indicate that the formulated drug (035-p18) has the independent function similar or better than commercial market product-Alendronate that is used for bone loss treatment. It is clearly to show that $(+) \mathrm{PMZ}$ has the significant anti-bone loss function, it is also suggested applying both $(+) \mathrm{PMZ}$ and Alendronate may produce the further improvement for bone loss.

\section{Acknowledgments}

This work was supported by Southwest Research project number: R8174.

\section{References}

[1] B. L. Riggs and L. J. Melton, III. The prevention and treatment of osteoporosis. N.Engl.J.Med. 327:620-627,8-27-1992.

[2] B. L. Riggs and L. J. Melton, III. The worldwide problem of osteoporosis: insights afforded by epidemiology. Bone. 17:505S-511S,1995.

[3] Grigoriev, V. S. Oganov, A. V. Bakulin, V. V. Poliakov, L. I. Voronin, V. V. Morgun, V. S. Shnaider, L. V. Murashko, V. E. Novikov, A. LeBlanc, and L. Shackelford. Clinical and Psychological Evaluation of Bone Changes Among Astronauts after Long Term Space Flights (Russian). Aviakosmicheskaia I Ekologicheskaia Meditsina. 32:21-25,1998.

[4] LeBlanc, V. Schneider, and L. Shackelford. Bone Mineral and Lean Tissue Loss after Long Duration Spaceflight. Trans.Amer.Soc.Bone Min.Res. 11S:567-1996.

[5] LeBlanc, L. Shackelford, A. Feiveson, and V. Oganov. Bone Loss in Space: Shuttle/Mir Experience and Bed-Rest Counter Measure Program. 1:17-1999.

[6] S. B. Arnaud. J. S. Harper, and M. Navidi. Mineral distribution in rat skeletons after exposure to a microgravity model.J.Gravit.Physiol. 2:115-116,1995.
[7] H. Uludag, T. Gao, G. R. Wohl, D. Kantoci, and R. F. Zernicke. Bone affinity of a bisphosphonate-conjugated protein in vivo. Biotechnol.Prog. 16:1115-1118,2000.

[8] H. Uludag and J. Yang. Targeting systemically administered proteins to bone by bisphosphonate conjugation. Biotechnol.Prog. 18:604-611,2002.

[9] J. E. Wright, L. Zhao, P. Choi, and H. Uludag. Simulating hydroxyapatite binding of bone-seeking bisphosphonates. Adv.Exp.Med.Biol. 553:139-148,2004.

[10] S.A. Gittens, G. Bansal, C. Kucharski, M. Borden, and H. Uludag. Imparting mineral affinity to fetuin by bisphosphonate conjugation: a comparison of three bisphosphonate conjugation schemes. Mol.Pharm. 2:392-406,2005

[11] S. Zhang, J. E. Wright, G. Bansal, P. Cho, and H. Uludag. Cleavage of disulfide-linked fetuin-bisphosphonate conjugates with three physiological thiols. Biomacromolecules. 6:2800-2808,2005.

[12] J. E. Wright, S. A. Gittens, G. Bansal, P. I. Kitov, D. Sindrey, C. Kucharski, and H. Uludag. A comparison of mineral affinity of bisphosphonate-protein conjugates constructed with disulfide and thioether linkages. Biomaterials. 27:769-784,2006.

[13] T.J. Hall, H. Nyugen, M. Schaeublin, M. Michalsky, and M. Missbach. Phenothiazines are potent inhibitors of osteoclastic bone resorption. Gen.Pharmacol. 27:845-848,1996.

[14] E. J. Boland, US patent application 2006/0258650 A1. Phenothiazine enantiomers as agents for the prevention of bone loss.2006

[15] D. P. Gallegos, Munn, K., Smith, D. M., and Stermer, D. L. A NMR Technique for the Analysis of Pore Structure: Application to Materials with Well-Defined Pore Structure, J. Colloid Interface Sci 119127.1987

[16] C.L.Glaves and Smith D.M. Membrane Pore Structure Analysis Via NMR Spin-Lattice Relaxation Experiments, J. Member. Sci. 46 167. 1989

[17] M.M. Chui, Philips, R.J., and McCarthy, M.Measurement of the Porous Microstructure of Hydrogels by Nuclear Magnetic Resonance, J. Colloid Interface Sci. 174336.1995

[18] W. E. Kenyon. Petrophysical Principles of Applications of NMR Logging, The Log Analyst, Mar.-Apr. 21.1997

[19] X. Wang, and Ni, Q. 2003. Determination of Cortical Bone Porosity and Pore size Distribution Using a Field NMR Approach, J. Orthop. Res. 21(2): 312-319.

[20] Q. Ni, King, J.D. and Wang, X.Characterization of Human Bone Structure Changes By Low Field NMR, Measurement Science and Technology, 15, 58 - 66. 2004.

[21] P. Fantazzini, Bortolotti, V., Brown, R.J.S., Garavaglia, C., Viola, R., Giavaresi, G.Two 1H-nuclear magnetic resonance methods to measure internal porosity of bone trabeculae: By solid-liquid signal separation and by longitudinal relaxation. Journal of Applied Physics Vol 95, 339-343. 2004.

[22] Q. Ni, and Nicolella, D.P. The Characterization of Human Cortical Bone Microdamage by Nuclear Magnetic 
Resonance. Measurement Science and Technology, 16, 659-668.2005

[23] Q. Ni, Nyman, J., Wang, X., De Los Santos, A. and Nicolella, D.Assessment of Water Distribution Changes in Human Cortical Bones by Nuclear Magnetic Resonance. Measurement Science and Technology, 18, 715-723.2007

[24] E. R. Morey-Holton, B. P. Halloran, L. P. Garetto, and S. B. Doty. Animal housing influences the response of bone to spaceflight in juvenile rats. J.Appl.Physiol. $88: 1303-1309,2000$

[25] H.Y. Carr, and Purcell, E. M. Effects of Diffusion on Free Precession in Nuclear Magnetic Resonance Experiments. Phys. Rev. 904, No.3 630. 1954
[26] S. Meiboom, and Gill, D. Modified Spin-Echo Method for Measuring Nuclear Relaxation Times. Rev. Sci. Inst. 29, 688. 1958

[27] K.R. Broenstein and C.E. Tarr. Importance of Classical Difussion in NMR Studies of Water in Biological Cells. Phys Rev. A 19, 2446, 1979.

[28] J. Kluge, F. Fusaro, N. Casas, M. Mazzotti, G. Muhrer. Production of PLGA micro- and nanocomposites by supercritical fluid extraction of emulsions: I. Encapsulation of lysozyme. The Journal of Supercritical Fluids, Volume 50, Issue 3, 327-335, 2009. 